\title{
Pulsed laser deposition of plasmonic nanostructured gold on flexible transparent polymer at atmospheric
}

\section{pressure}

\author{
Ronán McCann $^{1,2,3,4}$, Cian Hughes ${ }^{1,2,3}$, Komal Bagga ${ }^{1,2,3}$, Apryll Stalcup ${ }^{2,5}$, \\ Mercedes Vázquez ${ }^{1,2,5}$ and Dermot Brabazon ${ }^{1,2,3,4}$ \\ ${ }^{1}$ Advanced Processing Technology Research Centre, Dublin City University, \\ Glasnevin, Ireland \\ ${ }^{2}$ Irish Separation Science Cluster, National Centre for Sensor Research, Dublin City \\ University, Glasnevin, Ireland \\ ${ }^{3}$ School of Mechanical and Manufacturing Engineering, Dublin City University, \\ Glasnevin, Ireland; \\ ${ }^{4}$ National Centre for Plasma Science and Technology, Dublin City University, \\ Glasnevin, Ireland; \\ ${ }^{5}$ School of Chemical Sciences, Dublin City University, Glasnevin, Ireland \\ E-mail: komal.bagga@dcu.ie
}

\begin{abstract}
In this paper, we outline a novel technique for the deposition of nanostructured thin films utilizing a modified form of pulsed laser deposition (PLD). We demonstrate Confined Atmospheric PLD (CAP) for the deposition of gold on cyclic olefin polymer substrates. The deposition process is a simplified form of conventional PLD, with deposition conducted under atmospheric conditions and the substrate and target in close proximity. It was found that this confinement results in the deposition of nanostructured thin films on the substrate. Infrared spectroscopy showed no significant change of polymer surface chemistry as a result of the deposition process, and optical spectroscopy revealed plasmonic behavior of the resulting thin film. The effect of laser fluence on the deposition process was also examined with more uniform films deposited at higher fluences.
\end{abstract}

\section{Introduction}

Nanomaterial and nanostructured thin films have become of increasing interest in recent years. However, one roadblock to their full-scale adoption is the ability to deposit these nanomaterials on large area substrates. In addition to conventional deposition techniques such as plasma-enhanced chemical vapor deposition (PE-CVD) [1] and spray coating [2], pulsed laser deposition (PLD) has demonstrated promising potential for large area nanomaterial deposition. 
PLD is a mature technique for the creation of nanostructured thin and ultrathin films [3] with control of parameters such as plasma density and electron temperature during deposition having a large influence over the characteristics of resultant deposited material [4]. This control can be achieved through laser parameters such as fluence [4], wavelength [5] or pulse repetition frequency [6] or through process parameters such as target-to-substrate distance [7] or background gas pressure [8]. When conducted in vacuum or with a low-pressure background gas, stoichiometric transfer of material from the target to the substrate occurs forming a solid film upon deposition.

Ablation using an ultrashort femtosecond (fs) pulsed laser irradiation can generate nanoparticles within the plume, with the mechanism of nanoparticle formation being fragmentation and decomposition of the target material due to rapid heating [9]. The ultrafast nature of fs laser ablation produces a cooler and less dense plasma due to reduced laser-plume interaction when compared to picosecond or nanosecond ablation [10]. Plume velocities are also much greater in fs ablations resulting in the ability to deposit material prior to in-plume aggregation occurring [10]. The use of fs ablation has been exploited via Laser-Induced Reverse Transfer which has been shown to be successful in the creation of metal and hybrid metal ultrathin films on transparent substrates $[11,12]$.

PLD at atmospheric pressures has also been achieved, and is conventionally performed by placing the target within several millimeters of, and at an acute angle with respect to the substrate with target, irradiation occurring parallel to the substrate [13]. This technique has been successful for the creation of nanostructured gold [14] and diamond-like carbon [15] films.

Another successful variant of PLD for nanostructured film creation is Confined PLD which is achieved through spatially confining the expansion of the plasma plume by placing a transparent substrate in close proximity (approximately $50 \mu \mathrm{m}$ ) to the target, with target illumination occurring through the substrate. This proximity confines the plume in the direction of travel, sustaining a higher plasma density and election temperature at the deposition site with the plume condensing in the form of a nanomaterial film [16]. To date, this technique has been reported only for low pressure depositions.

In this paper, we outline a new variant of PLD, which we term "Confined Atmospheric PLD" (CAP), whereby Confined PLD is demonstrated for the first time at ambient atmospheric pressures using a picosecond pulsed solid-state laser. This technique is demonstrated for the deposition of nanostructured gold thin films, with the resultant film analyzed in terms of the film's morphology, optical properties, surface chemistry and crystalline structure.

\section{Experimental Method}




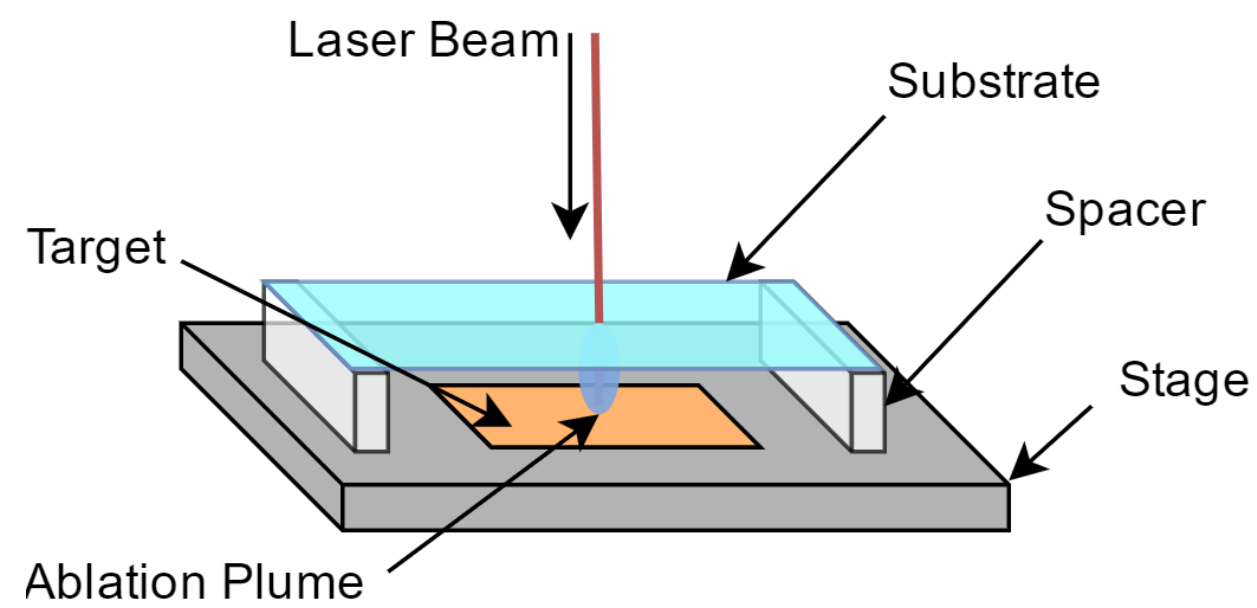

Figure 1. Schematic of the confined atmospheric pulsed laser deposition setup.

\subsection{Laser Deposition Setup}

The ablations were performed utilizing a picosecond-pulsed diode-pumped Wedge HF 1064 Nd:YAG laser (BrightSolutions, Italy). Specifications of the laser system used are outlined in Table 1. The experimental setup is a simplified form of conventional PLD whereby a transparent substrate is placed either in direct contact with a metallic target or with a spacer in between the target and substrate (figure 1), with the ablation performed under ambient atmospheric conditions. For the results presented here, the target-to-substrate distance was $50 \mu \mathrm{m}$ with the substrate fixed in place mechanically for the duration of processing.

Table 1. Specifications of the laser system used.

\begin{tabular}{lc}
\hline Wavelength & $1064 \mathrm{~nm}$ \\
Repetition Rate & $10 \mathrm{kHz}$ \\
Pulse Width & $700 \mathrm{ps}$ \\
Pulse Energy & $<160 \mu \mathrm{J}$ \\
Beam Diameter & $140 \mu \mathrm{m}$ \\
Fluence & $<1 \mathrm{~J} / \mathrm{cm}^{2}$ \\
\hline
\end{tabular}

For the experiments performed in this study, the laser beam was focused to a spot diameter of $140 \mu \mathrm{m}$ and unidirectionally rastered using an 2D galvanometer (Raylase SS-12) at a rate of 10 $\mathrm{mm} / \mathrm{s}$ across the target, mapping out a $5 \times 5 \mathrm{~mm}$ area in parallel lines with a $50 \mu \mathrm{m}$ step between each successive scan. The galvanometer was fitted with an $f$-theta lens with an effective focal length of 108 $\mathrm{mm}$. The current experimental setup allows for depositions over areas up to $100 \mathrm{~cm}^{2}$ but can be easily increased through alteration of the optical setup of the laser. To note, this process is differentiated 
from the similar technique Laser Induced Reverse Transfer by the use of longer laser pulse widths allowing for increased plume/laser interaction.

\subsection{Materials}

The target material was $99.9 \%$ metals basis gold foil (Agar Scientific, UK) with a thickness of 100 $\mu \mathrm{m}$. The substrate material was $188 \mu \mathrm{m}$ thickness ZeonorFilm ZF14-188 (Zeon Chemical L.P., Japan) cyclic olefin polymer (COP). The laser beam is incident on the target though the substrate, with the resultant ablation plume travelling towards the substrate. This offers promising opportunity to rapidly deposit nanostructured materials on transparent thin substrates, with enhanced adaptability. The use of flexible substrates also allows for the process to be adapted for high volume roll-to-roll production.

\subsection{Substrate Metrology}

The metrology of the deposited nanomaterial was analysed using Scanning Electron Microscopy (SEM) with an Evo LS50 (Zeiss, Switzerland). The samples were carbon coated to ensure conductivity prior to analysis with an Scancoat Six (Edwards, United Kingdom). Changes to the optical properties of the COP after deposition was examined using a Cary 50 UV-Vis-NIR spectrometer (Agilent, USA) scanning from $200-1100 \mathrm{~nm}$ at a rate of $600 \mathrm{~nm} / \mathrm{min}$. Attenuated Total Reflectance FourierTransform Infrared Spectroscopy (ATR-FTIR), which has a sampling depth up to $2 \mu \mathrm{m}$, was used to examine changes in the surface chemistry of the substrates after deposition using a Spectrum 100 (PerkinElmer, USA). Four scans were taken over $4000-600 \mathrm{~cm}^{-1}$ at resolution of $1 \mathrm{~cm}^{-1}$. The crystalline structure of the AuNPs was investigated using X-Ray Diffraction (XRD) using an AXS D8 x-ray diffractometer (Brucker, Germany).

\section{Results and Discussion}

\subsection{Surface Morphology}

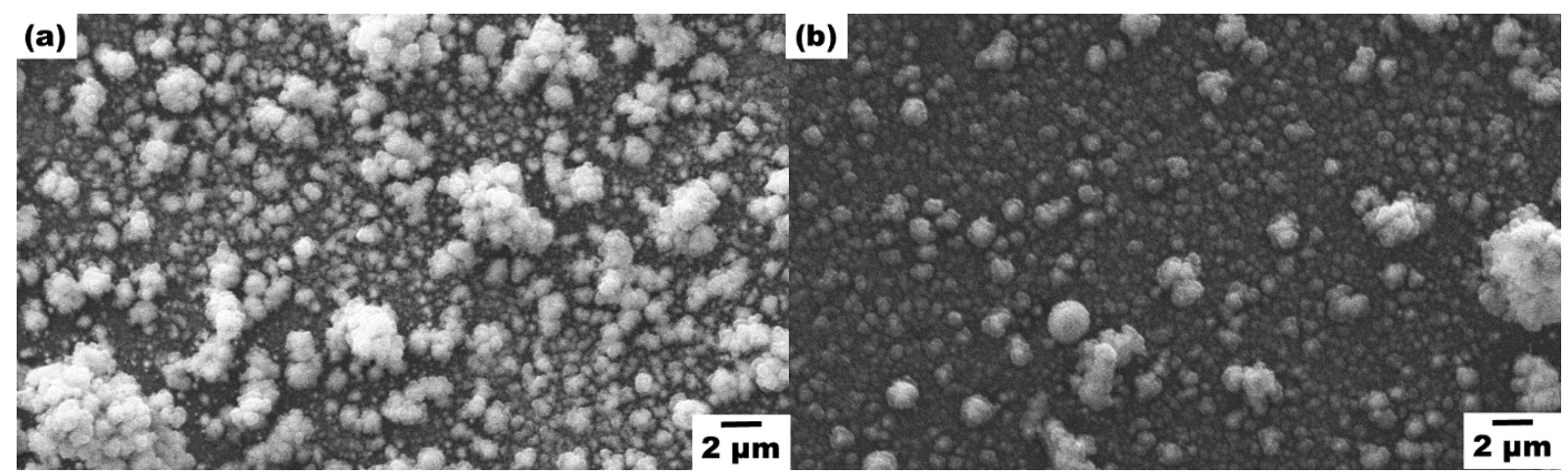

Figure 2. SEM images of gold nanoparticles deposited on COP via CAP using a laser fluence of (a) $0.22 \mathrm{~J} / \mathrm{cm}^{2}$ and (b) $0.48 \mathrm{~J} / \mathrm{cm}^{2}$. 
Figure 2 shows SEM images of gold nanoparticles (AuNPs) deposited on the COP substrates via CAP at laser fluences of $0.22 \mathrm{~J} / \mathrm{cm}^{2}$ and $0.48 \mathrm{~J} / \mathrm{cm}^{2}$. For the lower fluence depositions (figure 2a), a nanoparticle film was evident along with discreet nanostructured meso- and microparticle sized clusters. At higher fluence depositions (figure $2 b$ ), the nanomaterial film was seen to be more uniform with less micron-sized aggregated clusters than for lower fluence depositions.

As these larger nanostructured aggregate particles were less evident at the higher fluence depositions they are therefore likely a result of in-plume aggregation due to the colder plasma temperatures at lower ablation fluences [17]. Target fragmentation was ruled out as the mechanism for the microstructure formation, as at higher fluences larger ablated particulates would have sufficient kinetic energy to reach the substrate and would have been more preferentially seen as fluence increased. As seen for low-pressure confined PLD, the spatial confinement of the plume, by placing the substrate in close proximity of the target, allows for a high plasma temperatures which drives the formation of nanomaterial deposition at the substrate surface $[16,18]$. Through varying the target-tosubstrate distance and ablation fluence it may be possible to further reduce the size of the deposited nanoparticles.

As of yet, the upper limit of film thicknesses deposited via the CAP technique has not been found though work on gold films deposited via laser-induced reverse transfer found that the process window for deposition was quite small (at pulse energies from $36-40 \mathrm{~nJ}$ ) [12]. Depositions via the CAP technique were performed at larger pulse energies and over a larger range (up to $78 \mathrm{~mJ}$ ) allowing for higher material removal from the target as well as higher plume velocities, which may allow ultimately higher film thicknesses to be deposited. 


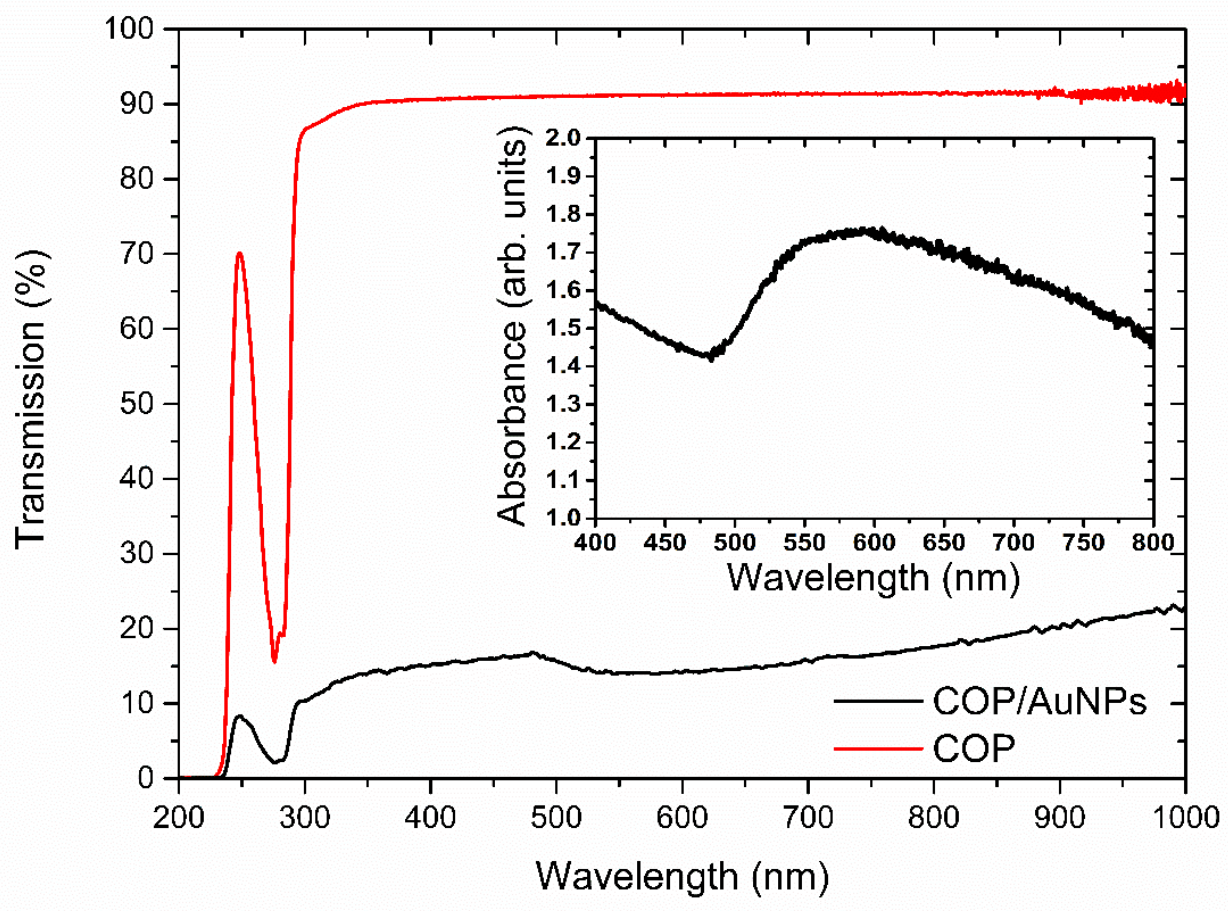

Figure 3. UV-Vis-NIR transmission spectra of the native COP substrate and COP after deposition of AuNPs with post-deposition absorbance spectrum from 400 - $800 \mathrm{~nm}$ (inset) highlighting the appearance of an absorbance band centered at $598 \mathrm{~nm}$.

\subsection{Optical Properties}

As native COP is noted for its high transparency from the NIR to Mid-UV wavelengths [19], UV-VisNIR transmission spectroscopy was performed to examine changes in the optical properties of the COP substrate after deposition. The transmission spectra of the COP before and after deposition is shown in figure 3. After deposition, a broadband decrease in transmission of between $10 \%-80 \%$ across all wavelengths was observed with this decrease becoming higher at longer wavelengths.

The plasmonic behavior of the film was also analyzed using UV-Vis-NIR absorbance spectroscopy. The appearance of a broad nature of an absorbance band after deposition, centered at $598 \mathrm{~nm}$ (inset figure 3), suggests a large nanoparticle size dispersion, while the largest portion of the band being towards longer wavelengths indicating the dispersity being weighted towards larger sized nanoparticles as evident from figure 2 [20]. The peak position of $598 \mathrm{~nm}$ is also indicative that the peak nanoparticle size is approximately $100 \mathrm{~nm}$ [21]. Tailoring of both the optical transmission and the plasmonic response of the thin film may possible through alteration of the thickness of the deposited material and the deposited nanoparticle size respectively. Through this it may also be 
possibly to remove the larger, aggregated particles with sizes outside of the nanoscale. This highlights the flexibility of the CAP process in the fabrication of optically active gold ultrathin films.

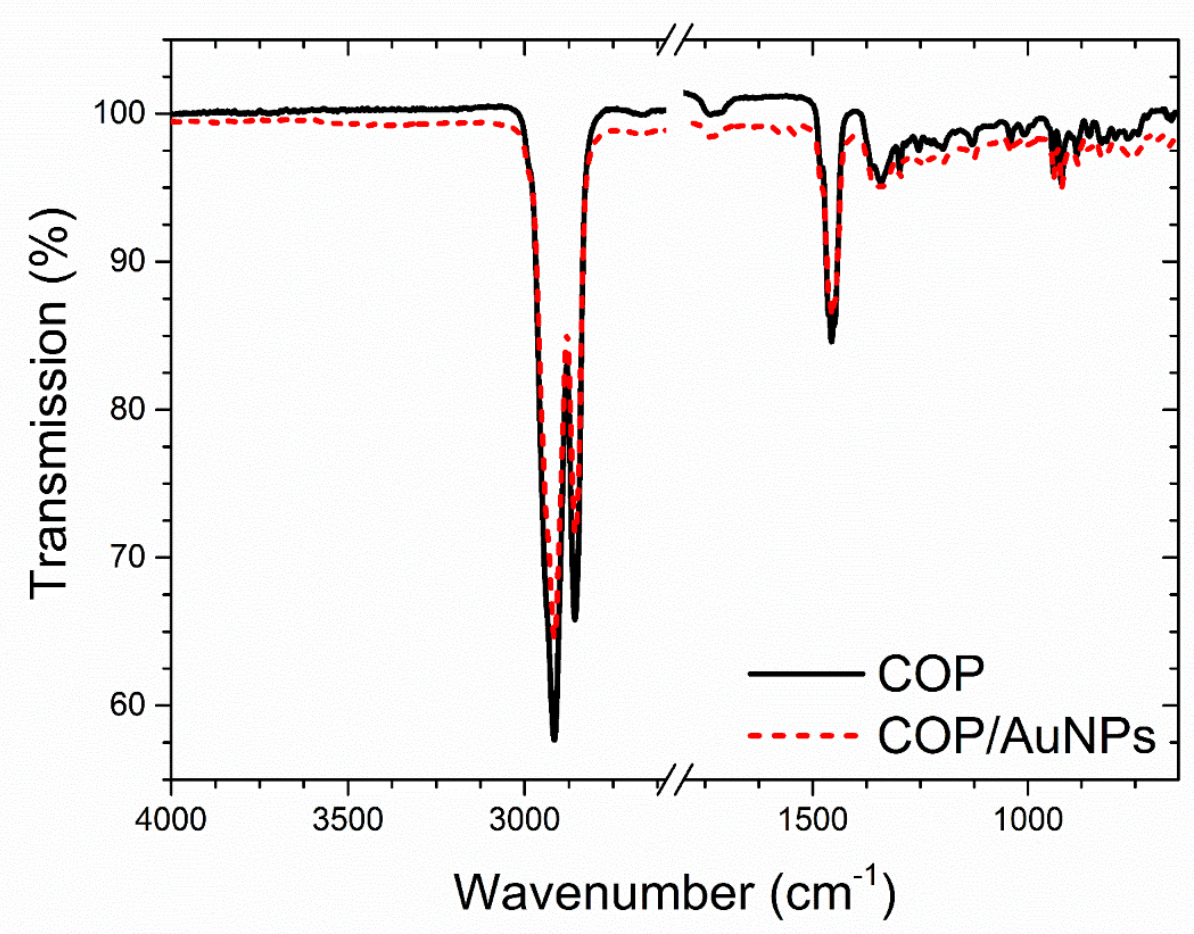

Figure 4. ATR-FTIR spectra of COP substrate and COP and after AuNP deposition at a fluence of $0.48 \mathrm{~J} / \mathrm{cm}^{2}$.

\subsection{Surface Chemistry}

Changes in the surface chemistry of the polymer film as a result of the laser deposition process were examined using ATR-FTIR spectroscopy (figure 4). Samples processed at the maximum fluence (0.48 $\mathrm{J} / \mathrm{cm}^{2}$ ) were chosen for analysis as any thermal degradation of the substrate would be more evident than when compared to lower fluence ablations.

Previous work on the thermal degradation of $\mathrm{COC}$ has seen the formation of spectral features such as increased $\mathrm{C}=\mathrm{O}$ stretching at $1710 \mathrm{~cm}^{-1}, \mathrm{C}=\mathrm{H}$ stretching $\left(2900-2800 \mathrm{~cm}^{-1}\right)$ and the formation of a large band at $4000-3000 \mathrm{~cm}^{-1}$ (indicative of $\mathrm{C}=\mathrm{C}-\mathrm{H}$ ) $[22,23]$. As the $\mathrm{C}=\mathrm{H}$ and $\mathrm{C}=\mathrm{O}$ bonds are at similar intensities before and after deposition, there has been limited oxidation and dehydrogenation of the surface due to the laser deposition process. Further evidence of this is no appearance of an increased $\mathrm{C}=\mathrm{C}$ signal at $1645-1445 \mathrm{~cm}^{-1}$ indicating no carbonization of the surface. Though some reduction in peak intensity was evident, this was attributed to the deposited gold film reducing the sampling depth into the COP bulk. Though no significant degradation was seen over the range of 
fluences examined in this work, previous studies have demonstrated substrate melting at fluences of $0.34 \mathrm{~J} / \mathrm{cm}^{2}$ and substrate warping at fluences of $0.79 \mathrm{~J} / \mathrm{cm}^{2}$ [23]. Neither warping or melting was evident from the morphological examination, however it is possible that damage may become evident if a larger laser fluence range is examined. It may be further possible to mitigate this damage through the use of focusing optics (allowing for a smaller beam waist at the target than the substrate) or through increasing target-to-substrate distance.

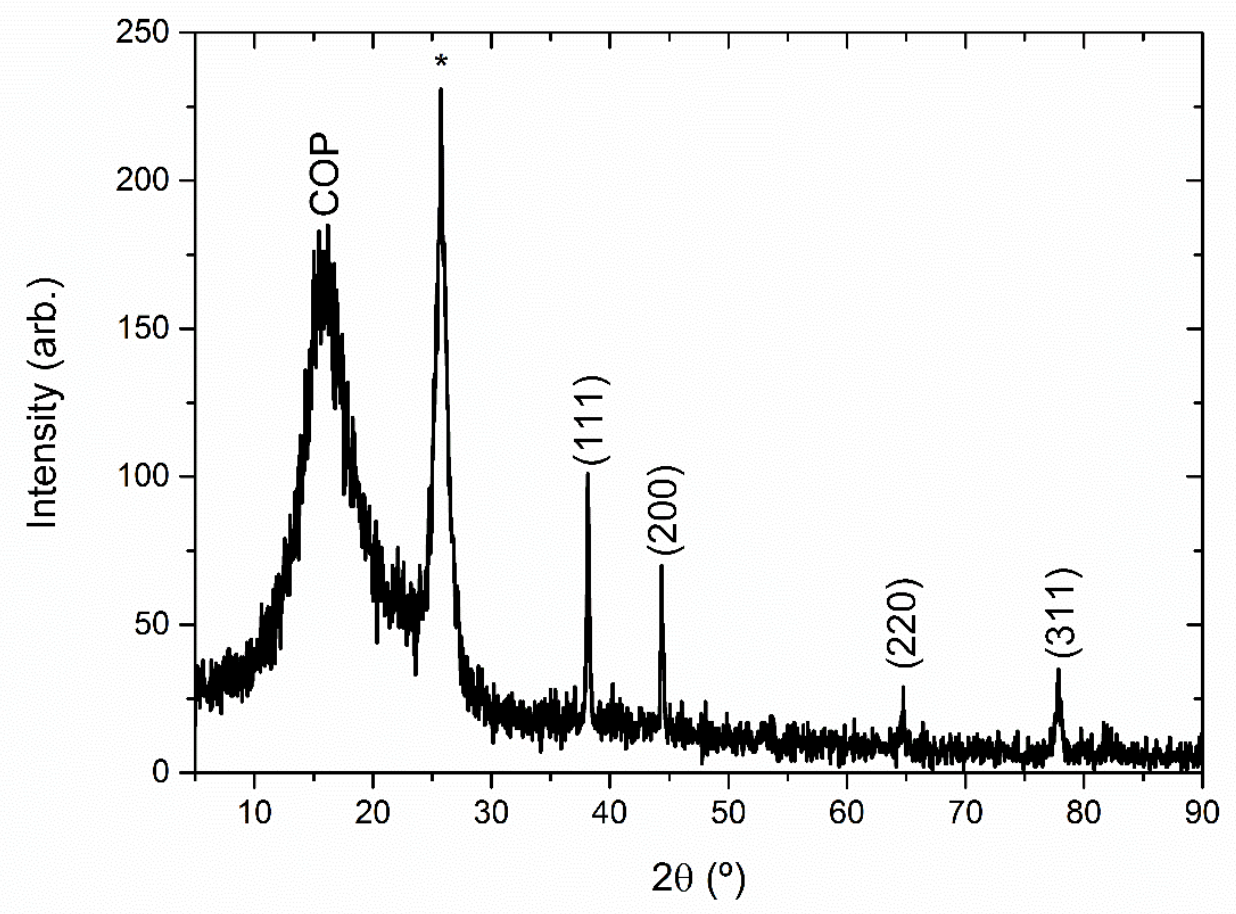

Figure 5. XRD spectrum of AuNPs deposited on a COP substrate with principle Bragg reflections labelled. The peak at $25^{\circ}$ was attributed to the adhesive used to affix the sample on the XRD stage.

\subsection{Crystalline Structure}

XRD analysis was performed to examine the crystalline structure of the substrate and deposited gold nanoparticles. A large broadband emission from the amorphous COP substrate from $10^{\circ}$ to $30^{\circ}$. The presence of four characteristic peaks corresponding to the (111), (200), (220) and (311) peaks of facecentered cubic gold are shown in figure 5. A ratio of 1.185 of the (200) and (111) peaks indicates that the nanoparticles are deposited in a random orientation. An average crystallite size of $6.60 \AA$ was estimated using the Scherrer equation [24]. The peak positions for the principle Bragg reflections are similar to that reported previously for AuNPs synthesized via wet chemical and biological techniques 
[25-27]. The peak at $25^{\circ}$ was attributed to the adhesive tape used to affix the sample within the XRD sample stage and is consistent with other data using the same method of XRD sample preparation [28].

\section{Conclusions}

In conclusion, the CAP process shows significant scope for the production of nanostructured thin films in ambient pressure conditions via laser ablation. Of specific interest is the ability for gold nanostructured thin films on polymer substrates. The polymer substrate showed no significant signs of degradation due to pulsed laser deposition, and the deposited gold film was seen to be plasmonically active. By varying the laser fluence, it was possible to alter the coverage and morphology of the deposited gold film. Though demonstrated for small areas in this work, when scaled, the process will allow for single step deposition of large area nanomaterial thin films on flexible substrates while being compatible with roll-to-roll processing for production scale-up. Future work towards optimization of the CAP process should lead to a simplified process for the fabrication of flexible electrodes suitable for chemical sensing and surfaces suitable for surface plasmon resonance spectroscopy.

\section{Acknowledgments}

The authors would like to thank Dr. Saikumar Inguvu for assistance with the XRD analysis. This publication has emanated from research supported by research grants from Science Foundation Ireland (SFI) under Grant Number 12/IA/1576 and the European Union's Horizon 2020 Research and Innovation Program under the Marie Sklodowska-Curie grant agreement No. 655194.

\section{References}

[1] Monaghan E, Michna T, Gaman C, O’Farrel D, Ryan K, Adley D, Perova T S, Drews B, Jaskot M and Ellingboe A R 2011 Characterisation of thin film silicon films deposited by plasma enhanced chemical vapour deposition at $162 \mathrm{MHz}$, using a large area, scalable, multitile-electrode plasma source Thin Solid Films 519 6884-6

[2] Scardaci V, Coull R, Lyons P E, Rickard D and Coleman J N 2011 Spray Deposition of Highly Transparent, Low-Resistance Networks of Silver Nanowires over Large Areas Small 7 $2621-8$

[3] Boyd I W 1996 Thin film growth by Pulsed Laser Deposition Ceram. Int. 22 429-34

[4] Escobar-Alarcón L, Arrieta A, Camps E, Romero S, Fernandez M and Haro-Poniatowski E 2008 Influence of the plasma parameters on the properties of aluminum oxide thin films deposited by laser ablation Appl. Phys. A 93 605-9

[5] Abdellatif G and Imam H 2002 A study of the laser plasma parameters at different laser wavelengths Spectrochim. Acta Part B At. Spectrosc. 57 1155-65 
[6] Guan L, Zhang D, Li X and Li Z 2008 Role of pulse repetition rate in film growth of pulsed laser deposition Nucl. Instruments Methods Phys. Res. Sect. B Beam Interact. with Mater. Atoms 266 57-62

[7] Galca A C, Stancu V, Husanu M A, Dragoi C, Gheorghe N G, Trupina L, Enculescu M and Vasile E 2011 Substrate-target distance dependence of structural and optical properties in case of $\mathrm{Pb}(\mathrm{Zr}, \mathrm{Ti}) \mathrm{O} 3$ films obtained by pulsed laser deposition Appl. Surf. Sci. 257 5938-43

[8] Fazio E, Neri F, Ossi P M, Santo N and Trusso S 2009 Growth process of nanostructured silver films pulsed laser ablated in high-pressure inert gas Appl. Surf. Sci. 255 9676-9

[9] Amoruso S, Bruzzese R, Wang X, Nedialkov N N and Atanasov P A 2007 Femtosecond laser ablation of nickel in vacuum J. Phys. D. Appl. Phys. 40 331-40

[10] Zeng X, Mao X L, Greif R and Russo R E 2005 Experimental investigation of ablation efficiency and plasma expansion during femtosecond and nanosecond laser ablation of silicon Appl. Phys. A 80 237-41

[11] Flury M and Pédri C 2013 Laser Induced Reverse Transfer with metal and hybrid material prepared with sol-gel process used on glass substrate Appl. Surf. Sci. 278 142-5

[12] Dhami G, Tan B and Venketakrishnan K 2011 Laser induced reverse transfer of gold thin film using femtosecond laser Opt. Lasers Eng. 49 866-9

[13] Steigert J, Haeberle S, Brenner T, Müller C, Steinert C P, Koltay P, Gottschlich N, Reinecke H, Rühe J, Zengerle R and Ducrée J 2007 Rapid prototyping of microfluidic chips in COC J. Micromechanics Microengineering 17 333-41

[14] Nedyalkov N, Nikolov A, Atanasov P, Alexandrov M, Terakawa M and Shimizu H 2014 Nanostructured Au film produced by pulsed laser deposition in air at atmospheric pressure $O p t$. Laser Technol. 64 41-5

[15] Kononenko T V, Konov V I, Lubnin E N and Dausinger F 2003 Pulsed laser deposition of hard carbon coatings at atmospheric pressure Quantum Electron.33 189-91

[16] Donnelly T and Lunney J G 2013 Confined laser ablation for single-shot nanoparticle deposition of silver Appl. Surf. Sci. 282 133-7

[17] Gamaly E G, Madsen N R, Rode A V. and Goldberg D 2010 Formation of Nanoclusters in Expanding Laser Plume Int. J. Nanosci. 9 371-5

[18] Gao X, Liu L, Song C and Lin J 2015 The role of spatial confinement on nanosecond YAG laser-induced Cu plasma J. Phys. D. Appl. Phys. 48175205

[19] Khanarian G and Celanese H 2001 Optical properties of cyclic olefin copolymers Opt. Eng. 40 1024

[20] Longobucco G, Fasano G, Zharnikov M, Bergamini L, Corni S and Rampi M A 2014 High 
stability and sensitivity of gold nano-islands for localized surface plasmon spectroscopy: Role of solvent viscosity and morphology Sensors Actuators B Chem. 191 356-63

[21] Haiss W, Thanh N T K, Aveyard J and Fernig D G 2007 Determination of Size and Concentration of Gold Nanoparticles from UV-Vis Spectra Anal. Chem. 79 4215-21

[22] Suriano R, Kuznetsov A, Eaton S M, Kiyan R, Cerullo G, Osellame R, Chichkov B N, Levi M and Turri S 2011 Femtosecond laser ablation of polymeric substrates for the fabrication of microfluidic channels Appl. Surf. Sci. 257 6243-50

[23] McCann R, Bagga K, Groarke R, Stalcup A, Vázquez M and Brabazon D 2016 Microchannel fabrication on cyclic olefin polymer substrates via $1064 \mathrm{~nm} \mathrm{Nd:YAG} \mathrm{laser} \mathrm{ablation} \mathrm{Appl.} \mathrm{Surf.}$ Sci. $387603-8$

[24] Mote V D, Purushotham Y and Dole B N 2012 Hall analysis in estimation of lattice strain in nanometer-sized $\mathrm{ZnO}$ particles J. Theor. Appl. Phys. 66

[25] Narayanan K B and Sakthivel N 2008 Coriander leaf mediated biosynthesis of gold nanoparticles Mater. Lett. 62 4588-90

[26] Chen Y, Gu X, Nie C-G, Jiang Z-Y, Xie Z-X and Lin C-J 2005 Shape controlled growth of gold nanoparticles by a solution synthesis Chem. Commun. 1044181

[27] Singaravelu G, Arockiamary J S, Kumar V G and Govindaraju K 2007 A novel extracellular synthesis of monodisperse gold nanoparticles using marine alga, Sargassum wightii Greville Colloids Surfaces B Biointerfaces 57 97-101

[28] Inguva S, Vijayaraghavan R K, McGlynn E and Mosnier J-P 2017 High quality interconnected core/shell $\mathrm{ZnO}$ nanorod architectures grown by pulsed laser deposition on $\mathrm{ZnO}$-seeded $\mathrm{Si}$ substrates Superlattices Microstruct. 101 8-14 